\title{
Impactos da tecnologia e do capital humano sobre o crescimento econômico asiático: uma abordagem via dados de painel
}

\author{
Élisson Telles Moreira*
}

\section{Resumo}

Este artigo examina os fatores de produção que obtiveram maior impacto sobre o crescimento do produto asiático no período de 1987-2004. Parte-se de uma amostra de 12 economias asiáticas. Para a estimação das equações, utilizou-se modelos econométricos, envolvendo dados de painel. Os resultados encontrados demonstram que o principal insumo de produção para o crescimento asiático foi a força de trabalho. Por fim, cabe ressaltar que a produtividade total dos fatores (PTF) tem um valor estacionário próximo a zero.

Palavras-chave: Crescimento econômico. Dados de painel. Produtividade. Capital humano.

"Quando vires um homem bom tenta imitá-lo, quando vires um homem mau, examina-te a ti mesmo.” Confúcio, Analectos, Sec. I A.C.

Economista. Mestre em Economia do Desenvolvimento. Professor da Universidade de Passo Fundo (UPF). E-mail: elisson@upf.br 


\section{Introdução}

Os países asiáticos, principalmente os localizados na região leste, têm demonstrado um forte crescimento do produto asiático nos últimos anos. Pode-se observar que países como Coréia do Sul, Cingapura e Taiwan obtiveram mudanças positivas em âmbito econômico e social ocasionadas, por este crescimento. Após o período da $2^{a}$ Guerra, essas nações evoluíram de uma situação inicial de baixos níveis de poupança doméstica, investimento e educação para elevados níveis desses insumos.

Inicialmente, em meados dos anos de 1990, o Banco Mundial ${ }^{2}$ formalizou um amplo estudo sobre as razões das altas taxas de crescimento das economias asiáticas. Este trabalho tinha como objetivo verificar se o crescimento desses países era provocado por acumulação, eficiência de alocação ou rápido catching-up tecnológico. Chegaram à conclusão que esse elevado crescimento era provocado pela eficiência no uso dos insumos. O relatório denominou esse fato como "milagre asiático". Essa denominação gerou vários debates na época. ${ }^{3}$ Alguns pesquisadores sociais, na maioria economistas, foram contrários a esse "milagre". Para eles, a condição inicial ${ }^{4}$ seria 0 fator central para o rápido crescimento asiático das décadas de 1960 a 1980.

Krugman (1994) foi um dos primeiros a analisar os resultados do estudo do Banco Mundial (BM). Ele informa que esse crescimento rápido foi promovido pela acumulação de insumos e não por ganhos de eficiência. Para desmistificar o "milagre", o autor lança a hipótese de retornos decrescentes de escala para os insumos. Dessa forma, chega-se num ponto em que os fatores de produção não contribuem mais para o crescimento do produto. $\mathrm{O}$ único fator que faz a economia crescer, numa situação dessas, é o progresso tecnológico (SOLOW, 1957).

Observa-se, nos estudos sobre o tema, dois fatos principais que corroboram com os modelos atuais de crescimento econômico. Primeiramente, a acumulação de insumos - o capital e o trabalho, entre outros - surge como o principal fator para o crescimento do produto. Assim, com o decorrer do tempo, as economias mais atrasadas tendem a aproximar-se - convergência - economicamente das mais desenvolvidas. O outro fato refere-se aos ganhos de eficiência auferidos por esses países, isto é, a melhor forma de utilização dos insumos - otimização - e o contínuo aprendizado dos trabalhadores (ARROW, 1962; LUCAS, 1988). A evidência empírica dos trabalhos sobre as economias do leste asiático apresenta os dois pontos de vista. Ora, favorecendo o Banco Mundial, ora contrariando os resultados do estudo.

Finalmente, cabe ressaltar que na atualidade, a discussão a respeito do crescimento asiático propõe verificar a relevância do progresso tecnológico e do capital humano em explanar as diferenças e as altas taxas de crescimento do produto per capita (COLLINS; BOSWORTH, 1996; SAREL, 1997; IWATA et al. 2002). Os motivos para essas análises são as desconfianças nos resultados obtidos por Young 
(1994; 1995), Kim e Lau (1994), entre outros. Portanto, essas novas análises veêm contribuir para o tema e lançam novas evidências e implicações.

A questão central deste artigo é identificar qual insumo impulsionou o crescimento econômico asiático no período de 1987 a 2004. Dessa forma, parte-se da análise de uma função de produção agregada - e as suas elasticidades - para avaliar o impacto dos insumos capital, trabalho, poupança, tecnologia e capital humano. Almeja-se, também, verificar a magnitude do impacto da crise asiática de 1997 e 1998 sobre o crescimento das economias da amostra.

$\mathrm{O}$ artigo divide-se em quatro partes. Inicialmente, apresenta-se uma análise do debate sobre o crescimento asiático. Nessa, levou-se em consideração a abordagem empírica sobre o crescimento asiático. Na segunda parte, tem-se o modelo econométrico e os dados. Em seguida, realiza-se a apresentação dos resultados. E, por fim, expõem-se as considerações finais da pesquisa.

\section{$2 \mathrm{O}$ debate sobre o crescimento econômico asiático}

As altas taxas de crescimento de um grupo específico de países asiáticos, a discussão da possibilidade da convergência - catching-up - desses países ao nível de bem-estar de países desenvolvidos e a questão da desigualdade - disparidade - são os principais temas de interesse no renascimento das pesquisas empíricas a respeito do crescimento econômico em fins da década de 1980 e início de 1990.

Pode-se inferir três fatores que induziram a estes novos estudos nesse período. Inicialmente, temos a formalização, em fins da década de 1980 de novas bases de dados, sendo a mais importante, a Penn World Tables, de Summer e Heston (1991), atualmente, com dados longitudinais de centenas de países. Segundo observa-se a preocupação dos países menos desenvolvidos em como auferir maiores taxas de crescimento econômico como um auxílio ao desenvolvimento econômico e social, assim sendo, suscita-se à questão da política econômica adequada a esses objetivos. E, por último, temos os novos modelos de crescimento econômico de acordo com Romer (1986; 1990), Lucas (1988), Grossman e Helpman (1991), e Aghion e Howitt (1992).

Duas questões intrigaram os pesquisadores: o rápido crescimento asiático é transitório ou haverá continuidade dessas altas taxas no longo prazo? O crescimento econômico asiático foi causado por simples acumulação de insumos ou pela produtividade total dos fatores (PTF)? Vejamos os desdobramentos e as implicações econômicas desses questionamentos à luz da teoria do crescimento econômico.

No caso da primeira questão, e de acordo com o modelo de Solow, em economias em que a renda per capita mais cresce são aquelas que apresentam um crescimento populacional mais expressivo, ceteris paribus. E também temos outro

Teoria e Evidência Econômica - Ano 20, n. 43, p. 213-237, jul./dez. 2014 
pressuposto que nos informa que no longo prazo, quanto maior for a taxa de poupança, maiores serão os produtos e o estoque de capital. Ora, isso se relacionas diretamente com a situação dos países asiáticos entre as décadas de 1950 e 1980, onde o crescimento populacional foi expressivo, o que levou à construção de um enorme estoque de mão de obra; e a taxa de poupança representa uma média de $35 \%$ do Produto Interno Bruto (PIB) nas economias do leste asiático (ADB, 2012). Assim, inicialmente, verifica-se que foi um Estado com orientação intervencionista que conduziu o processo, auxiliando, encorajando e planejando as economias desses países, em vista de um melhor padrão de vida no futuro - ideia que entra em conflito com a visão do Banco Mundial (1993) ao divulgar que o sucesso dessas economias deve-se ao livre mercado.

Lucas (1993) comenta que devemos olhar cada caso (país) de uma forma diferente -especialmente a Coréia do Sul. Se olharmos os países asiáticos sob a visão das teorias de crescimento endógeno - modelo de Lucas e modelo $\mathrm{AK}$ - há uma contraposição ao modelo tradicional de crescimento. Nos modelos em que a tecnologia é endógena, parte-se da suposição que a taxa de poupança impacta a taxa de crescimento equilibrado da economia ao admitirem que a acumulação de insumos não tenha retornos marginais decrescentes, mas sim, constantes, o que leva a um maior esforço de acumulação - poupança - e terá efeito permanente ao gerar uma maior taxa de crescimento. Assim, para essa teoria, são as disparidades nas taxas de investimento - ou poupança - dos países do leste asiático e as diferenças na taxa na qual as pessoas acumulam conhecimentos e habilidades, os fatores que levam a desigualdades permanentes nas taxas de crescimento.

Com referência ao segundo questionamento, e com base nos modelos de Lucas e AK, para explicar o crescimento econômico e os determinantes do progresso tecnológico dos países do leste asiático, elimina-se a noção de steady state, e, consequentemente, a convergência absoluta e relativa (BARRO, 1991). Assim, assume-se, para tanto, que os efeitos dos aumentos de capital e da força de trabalho sobre a tecnologia apresentam retornos em escala crescente, uma vez que parte-se da ideia que a acumulação de conhecimento é uma externalidade positiva. Dessa forma, os insumos têm retornos constantes e não decrescentes. Logo, um maior esforço de acumulação terá um efeito permanente de gerar uma maior taxa de crescimento de longo prazo, o que acarretará em um crescimento contínuo.

Neste artigo, parte-se da premissa que o debate tomou dois rumos, ambos com referência ao impacto do progresso tecnológico; o primeiro grupo, liderado pelo relatório do Banco Mundial (1993) argumenta que a PTF, com o passar dos anos, tem efeitos positivos e visíveis sobre as economias asiáticas; já o segundo grupo contradiz esses resultados (ver Quadro 1 e 2). Seus artigos e, principalmente, suas conclusões expõe que o crescimento asiático nas décadas de 1960 a 2000, foi causado por dois fatores 
principais: a acumulação de insumos, e.g., aumento na força de trabalho e educação; e a eficiência com que esses insumos são usados, o que, de fato, pode explicar o crescimento do produto. Na próxima seção, apresenta-se a metodologia do artigo.

Quadro 1 - Principais trabalhos empíricos - ênfase na hipótese de contribuição do progresso tecnológico sobre o crescimento

\begin{tabular}{|c|c|c|c|}
\hline Referência & Âmbito do estudo & Método & Conclusões \\
\hline $\begin{array}{l}\text { Young } \\
\text { (1992) }\end{array}$ & $\begin{array}{l}\text { Estudo comparati- } \\
\text { vo entre Hong Kong } \\
\text { e Cingapura. }\end{array}$ & $\begin{array}{l}\text { Índice translog do crescimento } \\
\text { da PTF de Gollop e Jorgenson } \\
\text { (1980) e Gollop, Jorgenson e } \\
\text { Fraumeni (1987). }\end{array}$ & $\begin{array}{l}\text { A PTF contribuiu de forma significativa para } \\
\text { o crescimento do produto de Hong Kong. } \\
\text { Já no caso de Cingapura o crescimento da } \\
\text { PTF foi instável - e levemente negativo - } \\
\text { influenciado pelo ciclo de negócios. }\end{array}$ \\
\hline $\begin{array}{l}\text { Banco } \\
\text { Mundial } \\
\text { (1993) }\end{array}$ & $\begin{array}{l}\text { Estudo sobre as } \\
\text { motivações para o } \\
\text { alto crescimento de } \\
\text { oito países do leste } \\
\text { asiático. }\end{array}$ & Regressões cross-country. & $\begin{array}{l}\text { A abertura da economia ao investimento } \\
\text { externo, as altas taxas de poupança, o alto } \\
\text { investimento em educação, os incentivos à } \\
\text { exportação, entre outros, levaram cresci- } \\
\text { mento "milagroso" desses países de } 1965 \\
\text { a } 1990 \text {. Em suma, o investimento privado } \\
\text { doméstico e o rápido crescimento em ca- } \\
\text { pital humano foram as principais alavancas } \\
\text { para o crescimento, em conjunto com uma } \\
\text { intervenção sistemática do Estado, esses } \\
\text { fatores contribuem com } 2 / 3 \text { do crescimento. }\end{array}$ \\
\hline $\begin{array}{l}\text { Collins e } \\
\text { Bosworth } \\
\text { (1996) }\end{array}$ & $\begin{array}{l}\text { Amostra de } 88 \text { pa- } \\
\text { íses, sendo oito do } \\
\text { leste asiático e cin- } \\
\text { co do sul da Ásia. }\end{array}$ & $\begin{array}{l}\text { Regressões cross-section com } \\
\text { funções de produção com dois } \\
\text { fatores, três fatores e fatores au- } \\
\text { mentados. }\end{array}$ & $\begin{array}{l}\text { A acumulação de capital físico contribuiu } \\
\text { com } 80 \% \text { do crescimento dos países do } \\
\text { leste asiático. A PTF teve contribuição mo- } \\
\text { desta. A hipótese é que o potencial de ado- } \\
\text { ção de tecnologias externas depende do } \\
\text { estágio de desenvolvimento de cada país. }\end{array}$ \\
\hline $\begin{array}{l}\text { Klenow e } \\
\text { Rodríguez- } \\
\text {-Clare } \\
(1997)\end{array}$ & $\begin{array}{l}\text { Amostra de dados } \\
\text { do painel de Sum- } \\
\text { mer e Heston com } \\
98 \text { países de } 1960- \\
1985 .\end{array}$ & $\begin{array}{l}\text { Regressões mincerianas para } \\
\text { o capital humano e função de } \\
\text { Cobb-Douglas. }\end{array}$ & $\begin{array}{l}\text { As diferenças no crescimento da PTF } \\
\text { explicam } 90 \% \text { da variação das taxas de } \\
\text { crescimento de produto por trabalhador. } \\
\text { Logo, evidencia-se a dominância do ca- } \\
\text { tch-up tecnológico e contraria-se os resul- } \\
\text { tados de Young }(1994,1995) \text {. }\end{array}$ \\
\hline $\begin{array}{l}\text { Sarel } \\
(1997)\end{array}$ & $\begin{array}{l}\text { Amostra de cinco } \\
\text { países da ASEAN } \\
\text { e os EUA e PWT } \\
\text { 5.6a. }\end{array}$ & $\begin{array}{l}\text { Estimação envolvendo três pas- } \\
\text { sos, cálculo das participações } \\
\text { de cada país em tecnologia, cál- } \\
\text { culo da intensidade relativa de } \\
\text { cada atividade econômica, para } \\
\text { cada país e cada ano a partici- } \\
\text { pação agregada da tecnologia. }\end{array}$ & $\begin{array}{l}\text { Os resultados do estudo indicam que } \\
\text { houve crescimento da PTF em Cingapura } \\
2,2 \% \text {, Tailândia } 2 \% \text { e Malásia } 2 \% \text { no perí- } \\
\text { odo de } 1978-96 \text {. }\end{array}$ \\
\hline $\begin{array}{l}\text { Easterly } \\
\text { e Levine } \\
\text { (2001) }\end{array}$ & $\begin{array}{l}\text { Penn World Tables } \\
\text { 5.6. }\end{array}$ & $\begin{array}{l}\text { Análise de trabalhos seminais } \\
\text { anteriores e regressões basea- } \\
\text { das no modelo de Mankiw, Ro- } \\
\text { mer e Weil (1992). }\end{array}$ & $\begin{array}{l}\text { Quando realizada uma análise compara- } \\
\text { tiva entre vários países, a PTF demons- } \\
\text { tra-se mais proeminente que a simples } \\
\text { acumulação de fatores na explicação do } \\
\text { crescimento do produto. }\end{array}$ \\
\hline $\begin{array}{l}\text { Iwata, } \\
\text { Kahn e } \\
\text { Murao } \\
\text { (2002) }\end{array}$ & $\begin{array}{l}\text { Amostra de nove } \\
\text { países do leste asi- } \\
\text { ático no período de } \\
1960-1990\end{array}$ & $\begin{array}{l}\text { Cross-section com modelo Neo- } \\
\text { clássico, função Cobb-Douglas, } \\
\text { regressão não paramétrica para } \\
\text { PTF. }\end{array}$ & $\begin{array}{l}\text { Crescimento similar do PTF entre os paí- } \\
\text { ses entre } 1960-1990 \text {. O capital físico con- } \\
\text { tribui com } 27 \% \text { do crescimento }\end{array}$ \\
\hline
\end{tabular}

Fonte: elaborado pelo autor (2014). 
Quadro 2 - Principais trabalhos empíricos - ênfase na hipótese de não contribuição do progresso tecnológico sobre o produto

\begin{tabular}{|c|c|c|c|}
\hline Referência & Âmbito do estudo & Método & Conclusões \\
\hline Tsao (1985) & $\begin{array}{l}\text { Amostra de } 28 \text { fir- } \\
\text { mas do setor manu- } \\
\text { fatureiro de Cinga- } \\
\text { pura no período de } \\
1970 \text { a } 1979 .\end{array}$ & $\begin{array}{l}\text { Função de produção trans- } \\
\text { log de Gollop e Jorgenson } \\
\text { (1980) para a decomposi- } \\
\text { ção da PTF. }\end{array}$ & $\begin{array}{l}\text { Crescimento da PTF foi muito baixa. } \\
\text { Os motivos podem ser o fato de a } \\
\text { maioria do capital ser estrangeiro, a } \\
\text { política de baixos salários e o influxo } \\
\text { de trabalhadores da Malásia. }\end{array}$ \\
\hline Kim e Lau (1994) & $\begin{array}{l}\text { Amostra de nove pa- } \\
\text { íses, sendo cinco de- } \\
\text { senvolvidos e quatro } \\
\text { "tigres". }\end{array}$ & $\begin{array}{l}\text { Função de meta-produção } \\
\text { agregada na forma translog } \\
\text { de Hayami e Ruttan (1970). }\end{array}$ & $\begin{array}{l}\text { Nos países desenvolvidos, o progres- } \\
\text { so tecnológico contribui } 46 \% \text { e } 72 \% \\
\text { para o crescimento. Já no caso dos } \\
\text { "tigres", não há contribuição - o prin- } \\
\text { cipal fator para esses é o capital entre } \\
48 \% \text { e } 72 \% \text {. }\end{array}$ \\
\hline Young (1994) & $\begin{array}{l}\text { Amostra de } 118 \text { pa- } \\
\text { íses da base de da- } \\
\text { dos de Summer e } \\
\text { Heston (1990). }\end{array}$ & $\begin{array}{l}\text { Regressão cross-section do } \\
\text { crescimento do produto por } \\
\text { trabalhador e do capital por } \\
\text { trabalhador. }\end{array}$ & $\begin{array}{l}\text { A acumulação rápida de capital e tra- } \\
\text { balho se apresenta como o principal } \\
\text { fator do crescimento. A PTF é irrele- } \\
\text { vante e está abaixo de países desen- } \\
\text { volvidos. Assim, não se sustenta o } \\
\text { argumento do Banco Mundial (1993) } \\
\text { sobre a orientação ao mercado ex- } \\
\text { terno como principal fator indutor de } \\
\text { crescimento. }\end{array}$ \\
\hline Young (1995) & $\begin{array}{l}\text { Amostra de quatro } \\
\text { países asiáticos. }\end{array}$ & $\begin{array}{l}\text { Função de produção trans- } \\
\log \text { para a decomposição } \\
\text { da PTF. }\end{array}$ & $\begin{array}{l}\text { O estudo demonstra que a PTF foi } \\
\text { de } 0,2 \% \text { em Cingapura, } 2,3 \% \text { Hong } \\
\text { Kong, } 1,7 \% \text { Coréia do Sul e } 2,1 \% \\
\text { Taiwan. O crescimento destes países } \\
\text { no período do pós-guerra foi resultan- } \\
\text { te de acumulação de fatores. }\end{array}$ \\
\hline Krugman (1994) & $\begin{array}{l}\text { "Tigres asiáticos", } \\
\text { Japão e China. }\end{array}$ & $\begin{array}{l}\text { Análise documental e com- } \\
\text { parativa dos trabalhos ante- } \\
\text { riores. }\end{array}$ & $\begin{array}{l}\text { O trabalho contradiz o argumento de } \\
\text { alta PTF nos países asiáticos do rela- } \\
\text { tório do Banco Mundial (1993). Rea- } \\
\text { lizando uma analogia com o caso da } \\
\text { URSS, demonstra-se que houve ape- } \\
\text { nas rápida acumulação e realocação } \\
\text { de fatores. }\end{array}$ \\
\hline Kim e Lau (1994) & $\begin{array}{l}\text { Amostra de nove pa- } \\
\text { íses, sendo cinco de- } \\
\text { senvolvidos e quatro } \\
\text { "tigres". }\end{array}$ & $\begin{array}{l}\text { Extensão do modelo ante- } \\
\text { rior com a inclusão do capi- } \\
\text { tal humano. }\end{array}$ & $\begin{array}{l}\text { Não encontram nenhuma contribui- } \\
\text { ção do progresso técnico. O cresci- } \\
\text { mento nesses países foi resultante de } \\
\text { crescimento em insumos tangíveis. }\end{array}$ \\
\hline Lau e Park (2003) & $\begin{array}{l}\text { Aumento da amostra } \\
\text { de Kim e Lau (1994) } \\
\text { com dados da déca- } \\
\text { da de } 1990 .\end{array}$ & $\begin{array}{l}\text { Função de meta-produção } \\
\text { agregada na forma translog } \\
\text { de Hayami e Ruttan (1970) } \\
\text { e regressão pooling time- } \\
\text {-series. }\end{array}$ & $\begin{array}{l}\text { A Coréia do Sul, Cingapura e Taiwan } \\
\text { elevaram os investimentos em P\&D; } \\
\text { porém, no geral, os resultados ainda } \\
\text { são rejeitados. A principal conquista } \\
\text { desses países é uma eficiente acu- } \\
\text { mulação de capital tangível. }\end{array}$ \\
\hline
\end{tabular}

Fonte: elaborado pelo autor (2014). 


\section{Metodologia de estudo e dados}

O referencial teórico utilizado constitui-se pelo método de decomposição de crescimento econômico endógeno. Este modelo transformou-se numa das mais úteis ferramentas para a verificação e análise de crescimento entre países. Pretende-se acrescentar a variável capital humano no estudo, como em Barro (1991), Lucas (1988) e Mankiw, Romer e Weil (1992).

A amostra de dados foi encontrada no Asian Development Bank (ADB), para o período de 1987-2004. O critério para a seleção do período foi a disponibilidade de dados macroeconômicos e, principalmente, as nações que obtiveram as mais altas taxas de crescimento econômico. Os dados utilizados no estudo foram a taxa de crescimento do PIB $(Y)$, a formação bruta de capital físico $(K)$, a força de trabalho $(L)$, proxies para o capital humano $(H)$ - taxa de alfabetização (pessoas acima de 15 anos de idade), utiliza-se de dados do relatório de desenvolvimento humano (2005) do Programa das Nações Unidas para o Desenvolvimento (PNUD) e o progresso tecnológico $(A)$ - utiliza-se os gastos com pesquisa e desenvolvimento $(\mathrm{P} \& \mathrm{D})$.

Para a escolha dos países, foi levada em consideração uma amostra de economias que fazem parte do chamado grupo dos "tigres asiáticos". Esses países são: Coréia do Sul, Taiwan, Cingapura, Hong Kong, Indonésia, Malásia, Filipinas, Tailândia, Mianmar; ${ }^{5}$ e, também, os países que apresentaram ascensão expressiva na sua economia, no período de estudo, são Índia, China e Vietnã.

\subsection{Modelo econométrico}

A função de produção a ser utilizada é a Cobb-Douglas com retornos constantes de escala. A seguinte função de produção agregada exibe o modelo matemático a ser utilizado:

$$
Y_{t}=A_{t} F\left(K_{t}, L_{t}, H_{t}\right)^{6}
$$

Onde $Y_{t}$ é o PIB real; $A_{t}$ a produtividade total dos fatores (PTF); $K_{t}$ o estoque de capital real; $L_{t}$ a força de trabalho; $H_{t}$ representa o estoque de capital humano. Com a logaritmização obtém-se:

$$
\log Y_{t}=\log A_{t}+\log K_{t}+\log L_{t}+\log H_{t}
$$


Primordialmente, a decomposição investiga o crescimento do produto como um componente que pode ser explicado pelo crescimento dos insumos. Analisa-se, também, um resíduo que captura as mudanças de produtividade.

\subsection{Modelo de regressão com dados de painel}

O modelo econométrico que estima o crescimento econômico é representado por dados de painel. Os motivos para a utilização de dados de painel constituem-se pelas vantagens que essa análise apresenta. Com dados de painel é possível controlar a heterogeneidade individual, ou seja, analisando especificamente as diferenças entre os países, os dados tornam-se mais informativos e menos colineares entre as variáveis (MÁTYÁS; SEVESTRE, 1996).

Essa estimação consiste de observações de $n$ variáveis para dois ou mais períodos de tempo $t$. Os países são indicados pelo subscrito $i$. O período de tempo para a análise inicia no ano de 1987 e termina em 2004, indicado pelo subscrito $t$.

No caso do presente estudo, representa-se como:

$$
\left(Y_{i t}, K_{i t}, L_{i t}, S_{i t}, A_{i t}, H_{i t}\right), i=1
$$

Onde:

$Y_{i t}$ é a taxa de crescimento do PIB real;

$K_{i t}$ é a taxa crescimento do estoque de capital (aproximado pela participação da formação bruta de capital fixo);

$L_{i t}$ é a taxa de crescimento da força de trabalho;

$S_{i t}$ é o nível da taxa de poupança em porcentagem;

$A_{i t}$ proxy do progresso tecnológico são os gastos com pesquisa e desenvolvimento (P\&D);

$H_{i t}$ proxy do estoque de capital humano é a taxa de alfabetização em porcentagem com idade acima de 15 anos;

\subsection{Modelo de regressão com Efeitos Fixos}

A regressão com Efeitos Fixos é um método para controlar variáveis em dados de painel quando essas variam entre os países, mas não ao longo do tempo. Essa regressão pode ser utilizada quando há duas ou mais observações temporais em cada país. 
O modelo de regressão com Efeitos Fixos tem $n$ interceptos diferentes, um para cada país. Esses podem ser representados por um conjunto de variáveis binárias, as Equais absorvem as influências de todas as variáveis omitidas que diferem de uma para outra, mas não são constantes ao longo do tempo.

A seguir, apresenta-se o modelo com a variável dependente (produto) e os regressores observados $(K, L, S, A, H)$ representados por $K_{i t}, L_{i t}, S_{i t}, A_{i t}$ e $H_{i t}$ :

$$
Y_{i t}=\beta_{0}+\beta_{1} K_{i t}+\beta_{2} L_{i t}+\beta_{3} S_{i t}+\beta_{4} A_{i t}+\beta_{5} H_{i t}+\beta_{6} Z_{i}+\varepsilon_{i t}
$$

Onde $Z_{i}$ é uma variável não observada, que varia de um país para outro, mas não ao longo do tempo. Deve-se estimar o impacto dos $\beta_{s}$ sobre o produto, mantendo constantes as características não observadas $Z$ de um país.

Como $Z_{i}$ varia de um país para outro, mas é constante ao longo do tempo, o modelo de regressão de crescimento do produto na equação (4) pode ser interpretado como sendo de $n$ interceptos, um para cada país. Particularmente, seja $\alpha=\beta_{0}+\beta_{5} Z_{i}$. Então, a equação (4) torna-se:

$$
Y_{i t}=\alpha_{i}+\gamma_{1} K_{i t}+\gamma_{2} L_{i t}+\gamma_{3} S_{i t}+\gamma_{4} A_{i t}+\gamma_{5} H_{i t}+\varepsilon_{i t}
$$

A equação (5) é o modelo de regressão com Efeitos Fixos, em que $\alpha_{1}, \ldots, \alpha_{n}$ são tratados como interceptos desconhecidos a serem estimados um para cada país. A interpretação de $\alpha_{i}$ como um intercepto específico para cada país na equação (4) vem de se considerar a reta de regressão do crescimento do produto para o $i$ - ésimo país. Essa reta é $\alpha_{i}+\beta_{1} K_{i t}+\beta_{2} K_{i t}+\beta_{3} S_{i t}+\beta_{4} A_{i t}+\beta_{5} H_{i t}$. O coeficiente de declividade da reta de regressão do crescimento do produto varia de um país para o outro. A fonte da variação do intercepto é a variável $Z_{i}$, a qual varia de um país para o outro, mas é constante ao longo do tempo.

Os interceptos específicos para cada país no modelo de regressão com Efeitos Fixos também fará uso de variáveis binárias para representar as particularidades de cada país. Como o estudo utiliza-se de uma amostra de 12 países, precisam-se incluir variáveis binárias adicionais para captar todos os interceptos específicos para cada país na equação (5).

Para desenvolver o modelo de regressão com Efeitos Fixos com variáveis binárias, seja $D 1_{i}$ uma variável binária igual a 1 quando $i=1$ e zero caso contrário; e $D 2_{i}$ igual a 1 quando $i=2$ e zero caso contrário, e sucessivamente. Não se pode incluir todas as $n$ variáveis binárias mais um intercepto comum, uma vez que, se for feito, os regressores serão perfeitamente colineares, de modo que se omite a 
variável $D 1$ para o primeiro grupo. Assim, o modelo de regressão com Efeitos Fixos na equação (5) pode ser escrito de forma equivalente como:

$$
Y_{i t}=\beta_{0}+\beta_{1} K_{i t}+\gamma_{2} D 2 \beta_{2} L_{i t}+\gamma_{3} D 3 \beta_{3} S_{i t}+\gamma_{4} D 4 \beta_{4} A_{i t}+\gamma_{5} D 5 \beta_{5} H_{i t}+\varepsilon_{i t}
$$

onde $\beta_{0}, \beta_{1} \ldots \gamma_{2}, \ldots, \gamma_{n}$ são coeficientes desconhecidos que serão estimados. Para derivar a relação entre os coeficientes na equação (4) e os interceptos na equação (5), comparam-se as retas de regressão do crescimento do produto nas duas equações. Na equação (5), a equação da regressão do crescimento do produto para primeiro país é $\beta_{0}+\beta_{1} K_{i t}+\beta_{2} L_{i t}+\beta_{3} S_{i t}+\beta_{4} A_{i t}+\beta_{5} H_{i t}$ e modo que $\alpha_{i}=\beta_{0} \alpha_{i}$. Para o segundo e os demais países, é $\beta_{0}+\beta_{1} K_{i t}+\beta_{2} L_{i t}+\beta_{3} S_{i t}+\beta_{4} A_{i t}+\beta_{5} H_{i t} \gamma_{i}$, de modo que $\alpha_{i}=\beta_{0}+\gamma_{i}$ para $i \geq 2$.

Para a estimação das equações (4) e (5) utiliza-se os mínimos quadrados ordinários (MQO) e os mínimos quadrados generalizados (MQG). O método de cálculo é feito em dois passos. No primeiro passo, a média específica do país é subtraída de cada variável. No segundo passo, a regressão é estimada, utilizando as variáveis com subtração da média do país. Dessa forma, pode-se demonstrar a transformação da equação (3) com o intuito de eliminar o efeito do componente não observado $u_{i}$

$$
\beta_{0}+\beta_{1} K_{i t}+\beta_{2} L_{i t}+\beta_{3} S_{i t}+\beta_{4} A_{i t}+\beta_{5} H_{i t}
$$

Onde:

$$
\widehat{Y}_{i}=T^{-1} \sum_{i=1}^{T} Y_{i t}, \widehat{K}_{i}=T^{-1} \sum_{i=1}^{T} K_{i t}, \hat{L}_{i}=T^{-1} \sum_{i=1}^{T} L_{i t}, \ldots, \hat{\varepsilon}_{i}=\hat{\varepsilon}_{i}=\sum_{i=1}^{T} \varepsilon_{i t}
$$

Assim, os $\beta_{s}$ podem ser estimados pela regressão de MQO das variáveis com subtração da média do grupo $\widehat{Y}_{i t}$ sobre $\widehat{K}_{i t}, \widehat{L}_{i t}, \hat{S}_{i t}$ entre outros.

$$
Y_{i t}-\hat{Y}_{i t}=\beta_{1}\left(K_{i t}-\widehat{K}_{i}\right)+\beta_{2}\left(L_{i t}-\hat{L}_{i}\right)+\beta_{3}\left(S_{i t}-\hat{S}_{i}\right)+\beta_{4}\left(A_{i t}-\hat{A}_{i}\right)+\beta_{5}\left(H_{i t}-\widehat{H}_{i}\right)+u_{i}+\hat{\varepsilon}_{i}
$$

O componente específico de cada país $u_{i}$ foi removido da equação (3). Com a estimação da equação (7) gera-se o estimador intragrupos (within). Já o estimador entre grupos (between) é obtido pela estimação da equação (6).

\subsection{Modelo de regressão com efeitos aleatórios}

O modelo de efeitos aleatórios assume que o intercepto $\alpha_{i}$ é uma variável aleatória. Esse não pode ser relacionado a $K_{i t}, L_{i t}$ e as outras variáveis, como também, ao $\varepsilon_{i t}$. Nesse caso, não se adicionam variáveis dummy. Assim, observa-se que: 


$$
Y_{i t}=\alpha_{i}+\beta K_{i t}+\beta L_{i t}+\beta S_{i t}+\beta A_{i t}+\beta H_{i t}+\varepsilon_{i t}
$$

$\mathrm{e}$

$$
\alpha_{i}=\alpha+u_{t}
$$

As inferências $E\left(\alpha_{i}\right)=\alpha, \operatorname{Var}\left(\alpha_{i}\right)=\sigma^{2} \alpha$ e $\operatorname{Cov}\left(\alpha_{i}, \varepsilon_{i t}\right)=0$ são assumidas independentes de $K_{i t}, L_{i t}$, entre outros. Reunindo as duas equações, (9) e (10), chega-se:

$$
Y_{i t}=\alpha_{i}+\beta K_{i t}+\beta L_{i t}+\beta S_{i t}+\beta A_{i t}+\beta H_{i t}+u_{t}+\varepsilon_{i t}
$$

O termo $u_{t}+\varepsilon_{i t}$ pode ser expresso resumidamente como $\phi_{i t}=u_{t}+\varepsilon_{i t}$. Assim, observa-se o novo modelo:

$$
Y_{i t}=\alpha_{i}+\beta K_{i t}+\beta L_{i t}+\beta S_{i t}+\beta A_{i t}+\beta H_{i t}+\phi_{i t}
$$

O termo estocástico $\phi_{i t}$ é composto de dois componentes: o erro $\varepsilon_{i t}$ e o erro específico individual $u_{t}$ o qual demonstra as particularidades de cada país. Esse termo varia com os países, no entanto é constante ao longo do tempo. O método para a estimação do modelo com efeitos aleatórios é o MQG.

\subsection{Teste de Hausman}

O teste de Hausman é feito para a averiguação de qual modelo de regressão estimado é mais eficiente e consistente. Assim, ele pode ser usado para testar a hipótese de desvios e inconsistências de um estimador. Se essa correlação não existe, o modelo de efeito aleatório é consistente e eficiente e o modelo de Efeitos Fixos é consistente, mas não é eficiente. No caso contrário, o estimador de Efeitos Fixos é consistente e eficiente, mas o estimador de efeitos aleatórios é inconsistente.

Assim, o teste de Hausman baseia-se no vetor de contrastes $\left(\hat{\beta}_{F E}-\hat{\beta}_{R E}\right)$. E para as estimativas das matrizes de variância e covariância dos modelos. Possui uma distribuição $\chi^{2} \operatorname{com} K$ graus de liberdade, sendo $K$ a dimensão do vetor de parâmetros. Ele é feito da seguinte forma:

$$
\xi H=\left(\hat{\beta}_{F E}-\hat{\beta}_{R E}\right)^{\prime}\left[\widehat{V}\left\{\beta_{F E}\right\}-\widehat{V}\left\{\beta_{R E}\right\}\right]^{-1}\left(\hat{\beta}_{F E}-\hat{\beta}_{R E}\right)
$$




\section{Resultados empíricos}

Inicialmente, foi utilizada uma transformação das variáveis em variações. Essa logaritmização visa eliminar tendências e indicar uma melhor robustez dos dados. A seguir, analisam-se os modelos estimados.

\subsection{Análise do modelo simples}

A taxa de acumulação - crescimento anual - de capital físico constitui-se um dos fatores centrais para a determinação do nível de produto real. Seus efeitos podem tornar-se mais longos dependendo da quantidade de inovação tecnológica incorporada ao novo capital. Para uma maior acumulação de capital, comprovadamente, é necessário uma determinada taxa de investimento e poupança.

As taxas de investimento, juntamente com o crescimento da força de trabalho, ajudam a determinar o caminho de crescimento de uma nação, logo, um aumento na taxa de investimento conduz o crescimento econômico a um novo patamar. E, também, dada uma taxa de investimento $x$, uma redução na taxa de crescimento da força de trabalho eleva o crescimento econômico.

Um aumento na taxa de investimento - ou uma queda na taxa de crescimento da força de trabalho - pode criar um período limitado de rápido crescimento da economia de um país, bem como uma elevação na taxa de investimento em pesquisa $\mathrm{e}$ desenvolvimento, comprovadamente, determina o crescimento econômico em longo prazo (ROMER, 1990). Um leve aumento no investimento $(I)$ e na poupança $(S)$ e uma redução da força de trabalho $(L)$ são consistentes com uma mudança positiva da taxa de crescimento e um temporário aumento na taxa de crescimento. $\mathrm{Na} \mathrm{Ta}-$ bela 1 demonstra-se as estimativas das regressões. O modelo 1 foi gerado a partir da equação 7. Primeiramente, incluiu-se, o parâmetro capital, força de trabalho e poupança. A variável dependente é o produto. O número de observações incluídas foi de 199 . 
Tabela 1 - Resultados da regressão 1987-2004

\begin{tabular}{|c|c|c|c|c|c|c|}
\hline \multicolumn{7}{|c|}{ Modelos de dados de painel: variável dependente - PIB real } \\
\hline \multirow{2}{*}{$\begin{array}{c}\text { Variáveis } \\
\text { independentes }\end{array}$} & \multicolumn{2}{|c|}{ Modelo simples } & \multicolumn{2}{|c|}{ Modelo intermediário } & \multicolumn{2}{|c|}{ Modelo completo } \\
\hline & $\begin{array}{c}F E \\
\text { (within) }\end{array}$ & $\begin{array}{c}R E \\
(G L S)\end{array}$ & $\begin{array}{c}F E \\
\text { (within) }\end{array}$ & $\begin{array}{c}R E \\
(G L S)\end{array}$ & $\begin{array}{c}F E \\
\text { (within) }\end{array}$ & $\begin{array}{c}R E \\
(G L S)\end{array}$ \\
\hline Ln L & $\begin{array}{r}0,530817^{\star} \\
(0,2390822)\end{array}$ & $\begin{array}{r}0,5548206^{*} \\
(0,23188)\end{array}$ & $\begin{array}{r}0,4845419^{*} \\
(0,2354646)\end{array}$ & $\begin{array}{r}0,5124741^{*} \\
(0,23188)\end{array}$ & $\begin{array}{r}0,4441229^{*} \\
(0,2353118)\end{array}$ & $\begin{array}{l}0,4951995 \\
(0,232837)\end{array}$ \\
\hline Ln K & $\begin{array}{r}0,205795^{\star \star} \\
(0,0409819)\end{array}$ & $\begin{array}{r}0,209428^{\star *} \\
(0,0392777)\end{array}$ & $\begin{array}{r}0,0113532^{* *} \\
(0,039706)\end{array}$ & $\begin{array}{l}0,0114416^{\star *} \\
(0,0392777)\end{array}$ & $\begin{array}{l}0,0069105^{\star *} \\
(0,0395689)\end{array}$ & $\begin{array}{r}0,0111666 \\
(0,0392647)\end{array}$ \\
\hline $\operatorname{Ln} S$ & $\begin{array}{r}0,1564732^{*} \\
(0,0435359)\end{array}$ & $\begin{array}{c}0,1616764^{*} \\
(0,0421478)\end{array}$ & $\begin{array}{r}0,1625859^{*} \\
(0,0428471)\end{array}$ & $\begin{array}{r}0,1635076^{*} \\
(0,0421478)\end{array}$ & $\begin{array}{r}0,156633^{*} \\
(0,0427473)\end{array}$ & $\begin{array}{r}0,162079 \\
(0,0421881)\end{array}$ \\
\hline $\operatorname{Ln} A$ & ---- & ---- & $\begin{array}{c}-0,0438758^{*} \\
(0,0169593)\end{array}$ & $\begin{array}{l}-0,0352326^{*} \\
(0,0122717)\end{array}$ & $\begin{array}{r}-0,0300619^{*} \\
(0,0186399)\end{array}$ & $\begin{array}{r}-0,0330766 \\
(0,0130718)\end{array}$ \\
\hline Ln H & --- & --- & --- & --- & $\begin{array}{l}-0,0055525^{\star} \\
(0,0031903)\end{array}$ & $\begin{array}{r}-0,0008822 \\
(0,0012387)\end{array}$ \\
\hline D Crise 1998 & $\begin{array}{c}-0,0269635^{\star} \\
(0,0157819)\end{array}$ & $\begin{array}{c}-0,0414673^{*} \\
(0,0114054)\end{array}$ & $\begin{array}{c}-0,0349254^{*} \\
(0,0115151)\end{array}$ & $\begin{array}{r}-0,0348564^{*} \\
(0,0114054)\end{array}$ & $\begin{array}{c}-0,0304571^{*} \\
(0,0117357)\end{array}$ & $\begin{array}{r}-0,0341349 \\
(0,0114454)\end{array}$ \\
\hline D Crise 2000 & $\begin{array}{c}-0,0416864^{*} \\
(0,0152319)\end{array}$ & --- & --- & --- & --- & ---- \\
\hline Constante & $\begin{array}{r}0,1230441^{*} \\
(0,078947)\end{array}$ & $\begin{array}{r}0,1232007^{*} \\
(0,0226762)\end{array}$ & $\begin{array}{r}0,1709595^{\star} \\
(0,020181)\end{array}$ & $\begin{array}{r}0,1613781^{*} \\
(0,0226762)\end{array}$ & $\begin{array}{r}0,6369184^{*} \\
(0,2684788)\end{array}$ & $\begin{array}{r}0,2356049 \\
(0,1061002)\end{array}$ \\
\hline \multicolumn{7}{|c|}{ Estatísticas } \\
\hline $\mathrm{R}^{2}$ & 0,1391 & 0,1391 & 0,1672 & 0,1661 & 0,1810 & 0,1703 \\
\hline Teste F & 5,88 & & 7,31 & & 6,66 & \\
\hline Wald chi & & 31,37 & & 40,32 & & 40,55 \\
\hline $\begin{array}{l}\text { Países } \\
\text { incluídos }\end{array}$ & 12 & 12 & 12 & 12 & 12 & 12 \\
\hline $\begin{array}{l}\text { Total de } \\
\text { observações } \\
\text { do painel }\end{array}$ & 199 & 199 & 199 & 199 & 199 & 199 \\
\hline
\end{tabular}

Fonte: elaboração do autor (2014).

Notas: ** $e^{*}$ denota significância e o nível de significância é de $1 \%$ e $5 \%$, respectivamente.

O modelo de Efeitos Fixos com a estimativa within, isto é, dentro dos países, adaptou-se melhor ao estudo. Os parâmetros estimados $\operatorname{LnK}, \operatorname{LnL}$ e $\operatorname{LnS}$ são equivalentes, respectivamente, a $\beta_{1}, \beta_{2}$ e $\beta_{3}$. Os valores estimados dos parâmetros foram de $\beta_{1}=0,0205, \beta_{2}=0,5308, \beta_{3}=0,1564$ e o intercepto $\alpha=0,1230$. 
Pode-se observar que os coeficientes estimados apresentam sinal esperado e são significativamente diferentes de zero, exceto o capital. Evidencia-se, portanto, que $o$ insumo força de trabalho tem efetivado uma influência preponderante sobre 0 crescimento asiático de 1987 a 2004. Logo, isso indica que uma variação de $1 \%$ na força de trabalho produz um impacto de aproximadamente $0,5 \%$ sobre o PIB.

Países como China, Índia, Indonésia, Tailândia e Vietnã tem uma força de trabalho numerosa. Nos anos de 1980 e 1990, os governos locais incentivaram às suas firmas a produzirem bens de baixo valor agregado e em larga escala. Os trabalhadores nesses países são, na maior parte, de origem rural; com o processo de industrialização da década de 1960 a década de 1980, houve uma elevação na demanda por mão de obra; assim, como uma forma de redução de custos, foi dada a preferência a esses trabalhadores. Na atualidade, especialmente em países com regimes autoritários, e.g., China e Vietnã, ainda persistem com baixos salários. Esse fato, aliado aos elevados investimentos públicos e incentivos à exportação, levou as firmas a visar o mercado externo, com exportações de produtos - eletroeletrônicos, brinquedos, têxteis. Assim, esses fatos corroboram para que a força de trabalho seja o principal fator de crescimento na Tabela 2.

O capital foi estimado por meio de uma proxy - formação bruta de capital fixo (FBCF) das economias, a qual significa a soma total de investimentos brutos (sem depreciação) em capital fixo (máquinas, edifícios entre outros) feitos por empresas estatais ou particulares em determinado período. Dessa forma, a proxy considera essa formação ou acumulação como um aumento ou diminuição de capital. O valor encontrado na estimativa foi de $\beta_{1}=0,0205\left(\sigma_{k}=0,0409\right)$. Evidencia-se, assim, que o capital tem pequena contribuição para o crescimento dos países da amostra.

A poupança pode ser conceituada como a renda não gasta pela força de trabalho. Existe uma relação de identidade entre a poupança e o investimento e, consequentemente, a formação bruta de capital fixo. Os investimentos asiáticos são garantidos por uma alta taxa de poupança interna. Desse modo, há uma dependência menor de capital externo. Posteriormente, com uma grande quantidade de poupança doméstica, uma economia pode fazer seus investimentos, além de sustentar o seu balanço de pagamentos. O parâmetro para a poupança revela um valor de $\beta_{3}=0,1564\left(\sigma_{s}=0,0435\right)$, o que demonstra o alto nível de poupança das economias asiáticas.

No período de estudo, 1987-2004, verifica-se o padrão de crescimento chamado de "gansos voadores". ${ }^{7}$ Esse modelo parte das sinergias entre um grupo de economias periféricas ao redor de uma economia mais desenvolvida. Nessa época, no continente asiático, foram feitos investimentos cruzados entre o Japão, a economia desenvolvida, e as nações compreendidas pelo leste, sul e sudeste asiático. Assim, 
as economias mais desenvolvidas foram repassando atividades com ênfase no trabalho para as outras nações asiáticas. Essas atividades requerem mão de obra barata, encontrada inicialmente em países como China, Índia, Vietnã e Filipinas.

A meta principal desse padrão de desenvolvimento industrial almeja conseguir uma determinada competitividade e diversidade nos negócios. Inicia-se, num primeiro momento, com o setor têxtil, segue com as indústrias químicas, siderúrgica e automotiva. Avança, posteriormente, para setores de maior valor agregado como eletroeletrônicos e tecnologia de informação. Cria-se, então, um ciclo de industrialização. ${ }^{8}$ Portanto, no caso asiático, via mão de obra barata, houve uma intensificação no insumo trabalho, fato evidenciado pelo alto valor do parâmetro estimado $\beta_{2}$.

Como o período de estudo compreende a crise asiática, para captar os efeitos da crise foram empregadas variáveis dummy. A crise asiática foi uma grande crise financeira com características, como: forte desvalorização da moeda em relação ao dólar, queda dos preços dos ativos acionários e fuga de capital. Apresentam-se duas análises para esse caso. Primeiramente, analisa-se o período de surgimento e expansão da crise. Esse período compreende os anos de 1997 a 1998. Na segunda forma, demonstram-se impactos da crise num espaço de tempo maior, assim, avalia-se o período de 1997 a 2000. O motivo para as duas análises é o de investigar se a crise asiática teve efeitos em um curto espaço de tempo ou durou mais tempo.

Os efeitos da crise sobre o crescimento econômico asiático são analisados pela equação 6 . Assim, seja $D 2_{i}$ uma variável binária igual a 1 quando $i=1$, para o caso de haver impactos da crise; caso contrário, igual à zero. Seja $D 2_{i}$ igual a 0 quando $i=0$ para o caso de não haver impactos da crise sobre o crescimento do produto.

Para o primeiro período, 1997 a 1998, o parâmetro estimado foi $D 2_{i}=-0,0269$ $\left(\sigma D_{97-98}=0,0157\right)$. Dessa forma, verifica-se que a crise asiática teve um impacto negativo sobre o crescimento do produto. O segundo período, 1997 a 2000, apresenta um parâmetro de $D 3_{i}=-0,0416\left(\sigma D_{97-00}=0,0152\right)$. O parâmetro mostra-se o dobro do anterior. Observa-se, então, que o impacto que a variável dummy capturou foi maior, ou seja, o efeito da crise asiática prolongou-se por um espaço de tempo maior.

Verifica-se que a crise asiática ocorreu num período em que a economia asiática demonstrava altos níveis de crescimento econômico, poupança e baixa inflação. Os únicos fatores negativos eram os mercados de ativos e os crescentes déficits em conta corrente (QUAH, 2008). Assim, aconteceram desvalorizações nas moedas nacionais e queda no preço de ativos financeiros. Consequentemente, houve uma fuga maciça de capitais especulativos. Isso levou a falência diversas instituições financeiras da Tailândia e de Hong Kong, entre outros países. 
Demonstra-se, a seguir, a regressão com efeitos aleatórios. Verifica-se que os parâmetros estimados são mais consistentes e eficientes que as estimativas da regressão de Efeitos Fixos.

\subsection{Análise do modelo intermediário}

O progresso tecnológico, a nível de países, pode ser avaliado de duas formas: pesquisa e desenvolvimento $(\mathrm{P} \& \mathrm{D})$ e difusão internacional de tecnologias. O primeiro pode ser considerado como um investimento que visa à obtenção de novos conhecimentos; o resultado do P\&D é demonstrado na forma de novas tecnologias, novos processos produtivos e organizacionais nas firmas e novos produtos. Já, o segundo, refere-se à disseminação - adoção - das tecnologias de países desenvolvidos pelos outros grupos de países. ${ }^{9}$ Dessa forma, na literatura econômica, atualmente, há um consenso a respeito do efeito positivo do progresso tecnológico para o crescimento das nações.

O P\&D pode ser influenciado pela iniciativa governamental ou empresarial. O Estado pode investir direta e indiretamente para o financiamento do P\&D, por meio de uma legislação favorável à inovação e subsídios às firmas inovadoras, entre outros. Esses financiamentos e auxílios são uma forma de incentivos em pesquisa; de forma concomitante, num país onde haja uma política eficiente de direitos de proteção intelectual e patentes, existem chances maiores para o incentivo à pesquisa privada.

Os pesquisadores em $\mathrm{P} \& \mathrm{D}$ - cientistas, engenheiros, entre outros - são pessoas treinadas para produzir novos conhecimentos - sejam esses simples ou complexos, inovações incrementais ou radicais. Isso requer altos níveis de educação, dedicação e persistência - algo inerente aos costumes, a cultura e a história asiática. ${ }^{10}$ Logo, as atividades de pesquisa tecnológica são mais comuns em países desenvolvidos, pois requer altos investimentos.

Observa-se que os gastos em $\mathrm{P} \& \mathrm{D}$ dos países asiáticos vêm crescendo conforme o passar dos anos. Isso reflete o interesse nessa área e o seu impacto no crescimento das nações. Todavia, enquanto o crescimento do produto foi expressivo, ao ponto de alguns economistas argumentarem que o seu modelo deveria ser aplicado aos países ocidentais, como foi verificado por Krugman (1994), as economias asiáticas não têm usufruído dos benefícios provenientes do progresso tecnológico.

A inovação tecnológica visa aumentar - e melhorar - a eficiência na produção de uma economia, dados os níveis de insumos disponíveis. Para mensurar o impacto do progresso tecnológico foram feitas duas regressões. A primeira envolve 
o modelo de Efeitos Fixos within estimada com a equação 7. Posteriormente, a segunda envolve o modelo de efeitos aleatórios estimada com a equação 10.

$\mathrm{O}$ modelo 2 aponta o valor estimado com a inclusão da variável tecnologia. $\mathrm{O}$ parâmetro estimado demonstra um valor de $\beta_{4}=-0,0438\left(\sigma_{\text {Ait }}=0,0169\right)$. Ele é significativamente diferente de zero e com sinal negativo. Isso implica que o progresso tecnológico ainda não contribuiu para os países do sudeste asiático. Uma resposta mais plausível é que o progresso tecnológico efetua-se com mais consistência em nações desenvolvidas. Dessa forma, as nações da amostra composta em sua maioria por países em desenvolvimento, não obtiveram ainda os impactos positivos do progresso tecnológico.

Já no caso da estimativa do modelo de regressão com efeitos aleatórios, o valor do parâmetro estimado para o progresso tecnológico foi de $\beta_{4}=-0,0352\left(\sigma_{\text {Ait }}=0,1227\right)$. Novamente, o sinal negativo está presente. Verifica-se que se aumentar em $1 \%$ os gastos em P\&D, eles terão um impacto de $0,02 \%$ negativos sobre o PIB.

\subsection{Análise do modelo completo}

O capital humano é um dos mais importantes insumos para o crescimento das nações, o qual assume que as novas habilidades, experiências e conhecimentos adquiridos são incorporados na força de trabalho como forma de capital humano. Dessa forma, ele pode ser considerado como um ativo em função do conhecimento e do talento humano.

Essa forma de capital está sujeita a noção de externalidades positivas, conforme o modelo de crescimento endógeno de Romer (1986) e Lucas (1988). Então, quanto mais treinada e habilitada estiver a força de trabalho, mais chances ela terá de obter um nível maior de renda no longo prazo. Assim, o investimento em capital humano pode ter um impacto positivo sobre o crescimento econômico dos países.

Pode-se argumentar que a educação e as novas ideias não têm contribuído para o crescimento asiático no período de estudo. Esse fato pode ser atribuído à extensão de tempo necessária para que o capital humano seja significativo e apresente resultados diretos na função de produção. No caso de países desenvolvidos, a educação da força de trabalho está à frente dos países em desenvolvimento. Assim, os resultados de uma melhor forma educacional refletem-se mais rápido, e de forma mais eficiente, em nações desenvolvidas do que nas em desenvolvimento.

Alguns economistas argumentam que as vantagens do capital humano não contribuem automaticamente para o crescimento (DURLAUF; JOHNSON; TEMPLE, 2004). A aquisição de novas ideias requer políticas específicas de investimento. 
Países como a Coréia do Sul e Cingapura tem um planejamento educacional exemplar. Esses países investiram maciçamente em educação e em projetos de alfabetização da população em meados das décadas de 1960 e 1970.

Na Tabela 1, o modelo 3 com a inclusão do capital humano demonstra a regressão com Efeitos Fixos e aleatórios. $O$ valor estimado para o parâmetro estimado $\beta_{5}$ revelou-se negativo e não é significativo ao nível de 5\% de significância. Trata-se de um resultado inesperado. Conforme os modelos de crescimento econômico, o capital humano apresenta um impacto positivo sobre o crescimento das nações. Contudo, conforme Kim e Lau (1994) pode-se inferir que o capital humano ainda não contribuiu para o crescimento dos países da amostra.

Por fim, por meio do teste de Hausman, temos indicações de que o modelo de regressão com efeitos aleatórios é mais significativo. $\mathrm{O}$ teste de Hausman para a comparação dos modelos ( $\xi H=3,48$ Prob $\chi^{2}=0,7466 \alpha=0,05$ não é significativo. Portanto, rejeita-se a hipótese nula de que os Efeitos Fixos sejam melhores.

\subsection{Análise da produtividade total dos fatores (PTF)}

Uma medida para o progresso tecnológico é a produtividade total dos fatores (PTF). Essa se caracteriza por ser uma tentativa de mensurar a produtividade, tomando para dentro do cálculo todos os fatores de produção. Desse modo, a produtividade está ligada ao conceito de função de produção agregada e refere-se a uma medida de eficiência $(A)$.

O método escolhido para o cálculo da PTF constitui-se pela função de produção agregada de Cobb-Douglas. Desse modo, a mudança técnica é vista como uma mudança da função de produção com o decorrer do tempo. A Figura 1 apresenta a decomposição da PTF por meio da observação dos resíduos da equação 7. Observa-se que os valores da produtividade são baixos e próximos de zero. Portanto, analisa-se que no período entre 1987 a 1995, a PTF apresentou um crescimento considerável. Contudo, no período posterior a 1995, verifica-se o início de um ciclo declinante da taxa de PTF. Isso pode ser atribuído ao fato da existência da crise asiática, 1997-1998, que teve impacto negativo nas economias asiáticas. 
Figura 1 - Produtividade total dos fatores para a amostra de países asiáticos 1987-2004

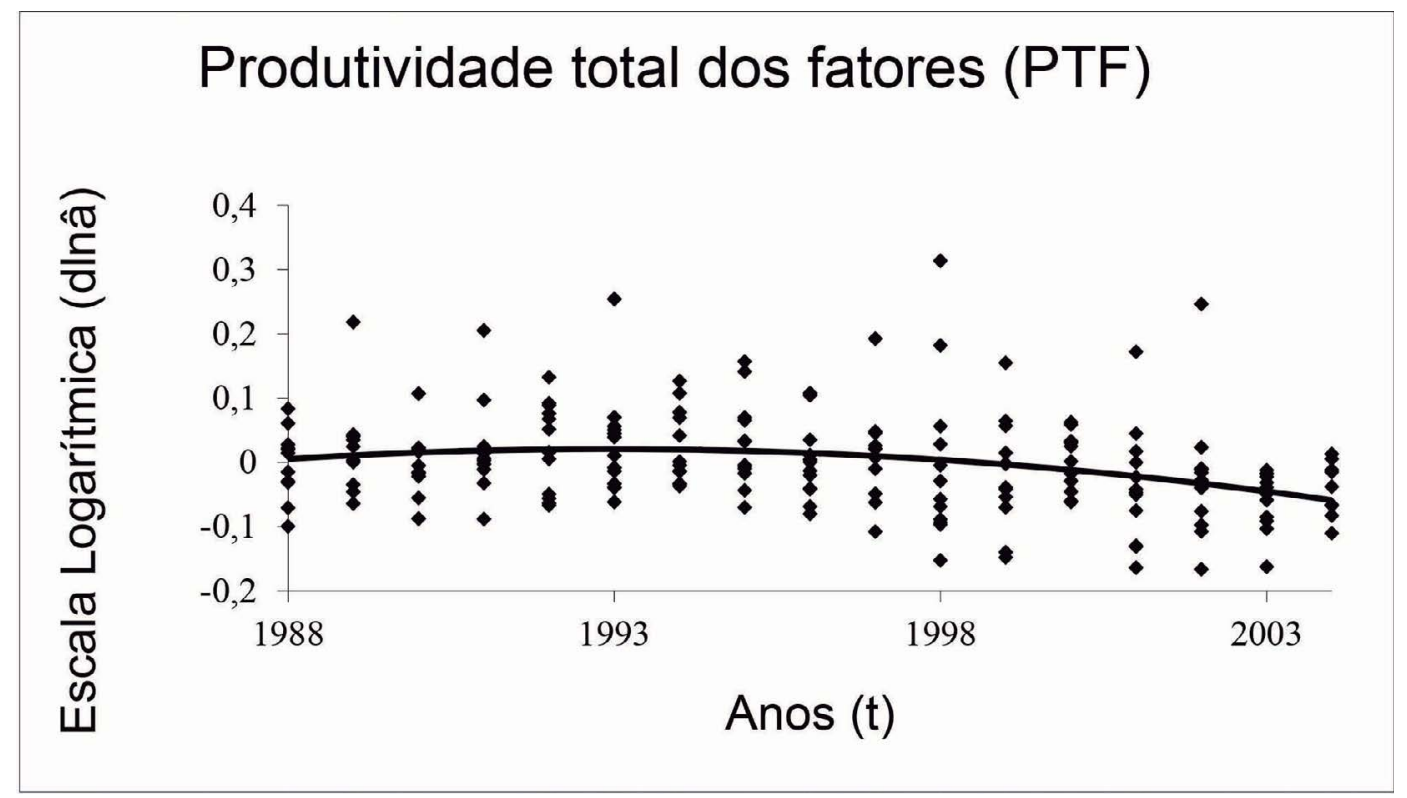

Fonte: elaborado pelo autor (2014).

\section{Considerações finais}

O objetivo principal deste artigo constituiu-se em averiguar os insumos de produção que obtiveram maior impacto sobre o crescimento do produto asiático no período de 1987-2004. Dessa forma, examinou-se uma amostra de 12 economias asiáticas. Avaliaram-se seis variáveis econômicas: o produto, o capital, o trabalho, a poupança, o progresso tecnológico e o capital humano. O modelo teórico utilizado foi o modelo neoclássico de crescimento econômico com a inclusão do capital humano. Para a estimação das equações utilizou-se de modelos econométricos, envolvendo dados de painel.

Os resultados encontrados demonstram que o principal insumo de produção para o crescimento asiático no período de estudo foi a força de trabalho. Esse fato colabora com as inferências de Young (1994) e Krugman (1994). O valor estimado para o capital mostrou-se de pequena significância. A proxy utilizada foi a formação bruta de capital fixo (FBCF). Nesse ponto, observa-se que a acumulação de fatores foi afetada pela crise asiática de 1997-1998 - fato averiguado por meio de uma variável dummy. Primeiramente, estimou-se o efeito da crise para os anos de 
1997 e 1998. Esse período mostrou-se significativo, porém o impacto da crise foi pequeno. Posteriormente, demonstrou-se a mesma análise para o período de 1997 a 2000. Verificou-se que o impacto da crise sobre o crescimento do produto foi negativo e significante a $5 \%$. Portanto, um período maior de tempo auferiu os impactos negativos da crise asiática.

O fator poupança demonstra um impacto positivo e significativo nas estimações. As altas taxas de poupança desses países durante o período de estudo ajudaram o crescimento do produto. Dessa forma, as economias asiáticas puderam aumentar o seu nível de investimento público.

Para a estimação dos insumos, do progresso tecnológico e do capital humano foram usados proxies. Essas se referem ao nível de pesquisa e desenvolvimento (P\&D) e taxa de alfabetização. Os resultados encontrados para os parâmetros foram de baixa significância e negativos. O parâmetro $A$, progresso tecnológico, é uma estimativa exógena do modelo de Solow. Assim, esse é informado como "resíduo" no modelo. Esse também é conhecido como produtividade total dos fatores. O parâmetro estimado foi $\beta_{4}=-0,0330$. Assim, observa-se que a produtividade total dos fatores (PTF) tem um valor estacionário próximo a zero. Os resultados encontrados corroboram com as análises fundamentais de Young (1994, 1995), Krugman (1994) e Kim e Lau (1994). A resposta plausível para esse fato é que os efeitos benéficos produzidos pelo progresso tecnológico e capital humano ainda não estão visíveis nessas economias.

Cabe ressaltar que o estudo envolveu uma análise de caráter exploratório. Foram encontradas dificuldades na obtenção de determinadas variáveis, e.g., capital humano. Deve-se constatar que a série de tempo encontrada é curta. Logo, foi necessária a utilização de dados de painel. Nesse sentido, existem muitos fatos relatados no estudo que podem ser discutidos de forma mais aberta e complexa. Sugere-se, como pesquisa futura, uma análise da difusão tecnológica dos países do leste asiático, na linha de pesquisa de Coe e Helpman (1995), Eaton e Kortum (2002), Keller (2004) e Benhabib e Spiegel (2005). Assim, abrem-se novos caminhos para a discussão do tema e suas principais implicações. 


\title{
Impacts of technology and human capital on asian economic growth: a panel data approach
}

\begin{abstract}
This paper presents a panel data methodology for understanding the Asian growth process. Growth accounting exercises show that the growth rates of the eighties and nineties are mainly due to the output per worker, while TFP growth is stationary around zero with no effects over long run growth. This exercise suggests that specific case, like "flying geese" perspective, play a key role in explaining the growth dynamics of the Asian experience in this period.
\end{abstract}

Keywords: Economic growth. Panel data. Productivity. Human capital.

Classificação JEL: O47; O53.

\section{Impactos de la tecnología y capital humano en el crecimiento económico de asia: un estudio por datos de panel}

\section{Resumen}

Este artículo examina los factores de producción que tuvieron el mayor impacto en el crecimiento del producto asiático en el período 1987-2004. Parte de una muestra es de doce economías asiáticas. Para la estimación de las ecuaciones, se utilizaron modelos econométricos que implican datos de panel. Los resultados muestran que lo principal input del crecimiento de Asia fue la fuerza de trabajo. Por último, hay que destacar que la productividad total de los factores (PTF) tiene un valor estacionario cerca de cero.

Palabras clave: Crecimiento económico. Datos de panel. Productividad. Capital humano. Classificacion JEL: O47; O53. 


\section{Notas}

1 Economista. Mestre em Economia do Desenvolvimento. Professor da Universidade de Passo Fundo (UPF). E-mail: elisson@upf.br.

2 The East Asian miracle: economic growth and public policy (1993).

3 Entre os quais, Amsden (1994), Lall (1994), Yanagihara (1994) e Kwon (1994).

4 Relaciona-se ao nível de insumos que uma economia possui em determinada época. No caso dos países asiáticos, nas décadas de 1960 a 1990, refere-se ao baixo nível de estoque de capital e alta taxa de poupança.

5 A literatura econômica, também, refere-se a esses 12 países como Newly Industrialized Countries (NIC). Essa categoria foi criada para designar países com altas taxas de crescimento econômico e industrialização a partir das décadas de 1960. No grupo de países identificados como NIC estão a Turquia, o Brasil, o México, a Argentina, o Chile e a África do Sul.

6 O modelo segue o critério de neutralidade de Hicks. Esse é um atributo de uma variável eficaz numa função de produção. $O$ atributo é que ele não afeta o trabalho diferentemente da forma com que ele afeta o capital. Ele é representado pelo parâmetro $A$. O principal exemplo constitui-se pela função de produção do modelo de Solow $Y=F(K, L)$. Onde $Y$ é o produto, $L$ o trabalho, $K$ o capital, $F$ a variável de produção e $A$ representa alguma forma de variável eficaz. $\operatorname{Em} Y=F(A K, L)$ a variável eficaz afeta o capital, mas não o trabalho. Em $Y=F(K, A L)$ ele afeta o trabalho, mas não o capital. Esses dois casos podem ser descritos como desvios de Hicks. Em $Y=A F(K, L)$ ele é Hicks-Neutro. Para mais detalhes ver Sala-i-Martin (2000).

7 Em inglês chama-se flying-gees" model, refere-se à forma de produção que foi introduzida, nos países menos desenvolvidos, por países desenvolvidos. Esse modelo descreve como a indústria passa por cinco estágios - introdução, substituição de exportações, exportação, maturação e fase de importação de bens primários. Esse modelo foi desenvolvido por Kaname Akamatsu em 1930. Para mais detalhes ver Korhonen (1994), Kojima (2000) e Kumagai (2008).

8 Esse padrão assemelha-se com a Teoria do Ciclo do Produto de Vernon (1966).

9 Para mais detalhes, ver Keller (2004) e Acemoglu (2009).

10 O componente cultural, especialmente o confucionismo, é um argumento central para o crescimento asiático. Logo, o confucionismo pode ser visto como um cruzamento entre a religião, um sistema de valores sobre a sociedade e uma ideologia de estado - que enfatiza a disciplina, a piedade, a hierarquia, a persistência, e, principalmente, a harmonia.

\section{Referências}

ACEMOGLU, D. Introduction to Modern Economic Growth Theory. New Jersey: Princeton University Press, 2009, 1009 p.

AGHION, P; HOWITT, P. A Model of Growth through Creative Destruction. Econometrica, v. 60, n. 2 (March., 1992), p. 323-51. 1992. Disponível em: <http://www.jstor.org/stable/2951599>. Acesso em: $16 \mathrm{dez} .2012$.

AMSDEN, A.H. Why Isn't the Whole World Experimenting with the East Asian Model to Develop?: Review of the East Asian Miracle. World Development, v. 22, n. 4, p. 627-33. 1994.

ARROW, K. J. The Implications of Learning by Doing. The Review of Economic Studies, v. 29, n. 3, (Jun., 1962), p. 155-173. 1962. Disponível em: <http://www.jstor.org/stable/2295952>. Acesso em: 17 dez. 2012.

ASIAN DEVELOPMENT BANK. Data and Research. 2012. Disponível em: <http://www.adb.org/ data/main>. Acesso em: 04 dez. 2012.

BANCO MUNDIAL. The East Asian miracle: economic growth and public policy. 1993. Disponível em: <http://tinyurl.com/yppcsq>. Acesso em: 4 dez. 2012. 
BARRO, R. J. Economic Growth in a Cross-Section of Countries. The Quarterly Journal of Economics, Vol. 106, Issue 2, pp. 407-443. 1991. Disponível em: <http://qje.oxfordjournals.org/content/106/2/407.abstract>. Acesso em: 16 dez. 2012.

BENHABIB, J; SPIEGEL, M. M. Human Capital and Technology Diffusion. In: AGHION, P; DULAUF, S. N. Handbook of Economic Growth. Amsterdan: Elsevier North-Holland, 2005.

COE, D.T; HELPMAN, E. International R\&D Spillovers. European Economic Review, v. 39, p. 859887. 1995. Disponível em:<http://www.sciencedirect.com/science/article/pii/001429219400100E >. Acesso em: 16 dez. 2012.

COLLINS, S. M; BOSWORTH, B. P. Economic Growth in East Asia: Accumulation versus Assimilation. Brookings Papers on Economic Activity, 2. 1996. Disponível em: <http://www.brookings. edu/ /media/Projects/BPEA/1996\%202/1996b_bpea_collins_bosworth_rodrik.PDF >. Acesso em: 16 dez. 2012.

DURLAUF, S. N; JOHNSON, P. A; TEMPLE, J. R.W. Growth Econometrics. 2004. Disponível em: <http://irving.vassar.edu/faculty/pj/growtheconometrics.pdf >. Acesso em: 07 dez. 2012.

EASTERLY, W; LEVINE, R. It's Not Factor Accumulation: Stylized Facts and Economic Growth. Disponível em: < http://papers.ssrn.com/sol3/papers.cfm?abstract_id=269108>. Acesso em: 16 dez. 2012.

EATON, J; KORTUM, S. Technology, Geography, and Trade. Econometrica, v. 70, n. 5, (Sep., 2002), p. 1741-1779. Disponível em: <http://www.jstor.org/stable/3082019>. Acesso em: 16 dez. 2012.

GROSSMAN, G.M; HELPMAN, E. Quality Ladders in the Theory of Growth. The Review of Economic Studies, v. 58, n. 1 (Jan., 1991), p. 43-61. 1991. Disponível em: <http://www.jstor.org/ stable/2298044>. Acesso em: 16 dez. 2012.

HUMAN DEVELOPMENT REPORT. International cooperation at a crossroads: Aid, trade and security in an unequal world. United Nations Development Programme. 2005. Disponível em: < http://hdr.undp.org/reports/global/2005/>. Acesso em: 16 dez. 2012.

IWATA, S; KHAN, S. M; MURAO, H. Sources of economic growth in East Asia: a non-parametric assessment. IMF Staff Papers, v. 50, n. 2, p. 157-177. 2003. Disponível em: <http://www.jstor.org/ stable/4149926 >. Acesso em 16 dez. 2012.

KELLER, W. International Technology Diffusion. Journal of Economic Literature, v. XLII (September 2004), p. 752-782. 2004. Disponível em: <http://spot.colorado.edu/ kellerw/ITD.pdf> Acesso em: 6 dez. 2012.

KIM, Jong-Il; LAU, L. J. The Sources of Economic Growth of the East Asian Newly Industrialized Countries. Journal of the Japanese and International Economies, v. 8, Issue 3, September 1994, p. 235-271. 1994. Disponível em: <http://www.sciencedirect.com/science/article/pii/ S0889158384710136>. Acesso em: 07 dez. 2012.

KLENOW, P; RODRÍGUEZ-CLARE, A. The Neoclassical Revival in Growth Economics: Has It Gone Too Far?. NBER Macroeconomics Annual 1997, Vol. 12. 1997. Disponível em: < http://www. nber.org/chapters/c11037.pdf> Acesso em: 16 dez. 2012.

KOJIMA, K. The "flying geese" model of Asian economic development: origin, theoretical extensions, and regional policy implications. Journal of Asian Economics, v. 11, p. 375-401. 2000. 
KORHONEN, P. The Theory of the Flying Geese Pattern of Development and Its Interpretations. Journal of Peace Research, local de publicação, v. 31, n. 1, p. 93-108, 1994

KRUGMAN, P. The Myth of Asia's Miracle. Foreign Affairs 73(6) Nov. /Dec. 1994, p. 62-78. Disponível em: < http://www.foreignaffairs.org/19941101faessay5151/paul-krugman/the-myth-of-asia-s-miracle.html> Acesso em: 7 dez. 2012.

KUMAGAI, S. A Journey Through the Secret History of the Flying Geese Model. IDE Discussion Paper No. 158. 2008.

KWON, J. The East Asia Challenge to Neoclassical Orthodoxy. World Development, v. 22, n. 4, p. 635-44. 1994.

LALL, S. The East Asian Miracle: Does the Bell Toll for Industrial Strategy? World Development, v. 22, n. 4, p. 645-54. 1994.

LAU, L.J; PARK, J. The Sources of East Asian Economic Growth Revisited. 2003. Disponível em: <http://www.stanford.edu/ ljlau/RecentWork/RecentWork/030921.pdf> Acesso em: 7 dez. 2012.

LUCAS, R. E. Jr. On the Mechanics of Economic Development. 1988. Disponível em: <http: /www. nber.org/papers/r1176>. Acesso em: 7 dez. 2012.

. Making a Miracle. Econometrica, v. 61, n. 2, (Mar., 1993), p. 251-272. 1993. Disponível em: <http://www.jstor.org/stable/2951551>. Acesso em: $16 \mathrm{dez} .2012$.

MANKIW, N. G; ROMER, D; WEIL, D. N. A Contribution to the Empirics of Economic Growth. The Quarterly Journal of Economics, v. 107, n. 2, May 1992, p. 407-437. Disponível em: <http:// qje.oxfordjournals.org/content/107/2/407.full.pdf >. Acesso em: 07 dez. 2012

MÁTYÁS, L; SEVESTRE, P. The Econometrics of Panel Data: A Handbook of Theory with Applications. Series: Advanced Studies in Theoretical and Applied Econometrics, v. 33. 2. ed., rev. ed., 1996, 948 p., Springer Netherlands.

QUAH, D. Post 1990s East Asian Economic Growth. In: ITO, T; HAHN, C.H. (Ed.). The Rise of China and Structural Changes in Korea and Asia, 2008.

ROMER, P. M. Increasing Returns and Long-run Growth. Journal of Political Economy, v. 94, n. 5, (Oct., 1986). 1986. Disponível em: <http://www.jstor.org/stable/1833190>. Acesso em: $07 \mathrm{dez}$. 2012.

Endogenous Technological Change. Journal of Political Economy, v. 98, n. 5, p. s71s102. 1990. Disponível em: < http://www.jstor.org/stable/2937632>. Acesso em: 07 dez. 2012.

SALA-I-MARTIN, X. Apuntes de Crecimiento Económico. 2. ed. Barcelona: Antoni Bosch, 2000.

SAREL, M. Growth and Productivity in ASEAN Countries. IMF Working Paper, No. 97/97. 1997. Disponível em: <http://www.imf.org/external/pubs/ft/wp/wp9797.pdf>. Acesso em: 16 dez. 2012.

SOLOW, R. M. A Contribution to the theory of Economic Growth. The Quarterly Journal of Economics, Vol. 70, No. 1, pp. 65-94. 1956. Disponível em: <http://qje.oxfordjournals.org/content/70/1/65.full.pdf+html >. Acesso em: 07 dez. 2012.

. Technical Change and The Aggregate Production Function. The Review of Economics and Statistics, v. 39, n. 3, (Aug., 1957), pp. 312-320. 1957. Disponível em: <http://www.jstor.org/ stable/1926047>. Acesso em: $16 \mathrm{dez} .2012$. 
SUMMER, R; HESTON, A. The Penn World Table (Mark 5): An Expanded Set of International Comparisons, 1950-1988. The Quarterly Journal of Economics, v. 106, n. 2, (May, 1991), p. 32768. 1991. Disponível em: < http://www.jstor.org/stable/2937941>. Acesso em: 16 dez. 2012.

TSAO, Y. Growth without Productivity: Singapore manufacturing in the 1970s. Journal of Development Economics, v. 18, p. 25-38. 1985. Disponível em: <http://www.sciencedirect.com/science/ article/pii/0304387885900379>. Acesso em: 16 dez. 2012.

VERNON, R. (1966). La inversión internacional y el comercio internacional en el ciclo de productos. In: Rosenberg, N. (org.). Economia Del Cambio tecnológico. Trad. de Eduardo L. Suárez. México: Fondo de Cultura Econômica, 1979. (espanhol), El trimestre Económico, Lecturas, 31, p. 408-427.

YANAGIHARA, T. Anything New in the Miracle Report? Yes and No. World Development, v. 22, n. 4, p. 663-70. 1994.

YOUNG, A. A Tales of Two Cities: Factor Accumulation and Technical Change in Hong Kong and Singapore. National Bureau of Economic Studies Macroeconomics Annual 1992, v. 7. Disponível em: <http://www.nber.org/chapters/c10990.pdf>. Acesso em: 16 dez. 2012.

Lessons from the East Asian NICS: A contrarian view. European Economic Review, v. 38, p. 964-73. 1994. Disponível em: <http://www.sciencedirect.com/science/article/ pii/0014292194901325 >. Acesso em: 16 dez. 2012.

. The Tyranny of Numbers: Confronting the Statistical Realities of the East Asian Growth Experience. The Quarterly Journal of Economics, v. 110, n. 3, (Aug., 1995), p. 641-680. 1995. Disponível em: <http://www.nber.org/papers/w4680>. Acesso em: 16 dez. 2012. 